7

\title{
Universal Gas-Uptake Behavior of a Zeolitic Imidazolate Framework ZIF-8 at High Pressure
}

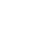

${ }^{1}$ Department of Earth System Sciences, Yonsei University, Seoul 03722, Republic of Korea

${ }^{2}$ Department of Chemistry, Soongsil University, Seoul 06978, Republic of Korea

${ }^{3}$ Department of ICMC Convergence Technology, Soongsil University, Seoul 06978, Republic of Korea

${ }^{4}$ NanoCenter and Department of Chemistry \& Biochemistry, University of South Carolina, Columbia, South Carolina 29208, United States

${ }^{5}$ Center for High Pressure Science \& Technology Advanced Research (HPSTAR), Shanghai 201203, China

Jinhyuk Choi, ${ }^{1}$ Junhyuck Im, ${ }^{1}$ Kyungkyou Noh, ${ }^{2}$ Jaheon Kim,,${ }^{2,3}$ Thomas Vogt, ${ }^{4}$ Yongjae Lee ${ }^{5, *}$ 


\section{Contents}

21 Part I. Supplementary Figures

22 Part II. Supplementary Tables 


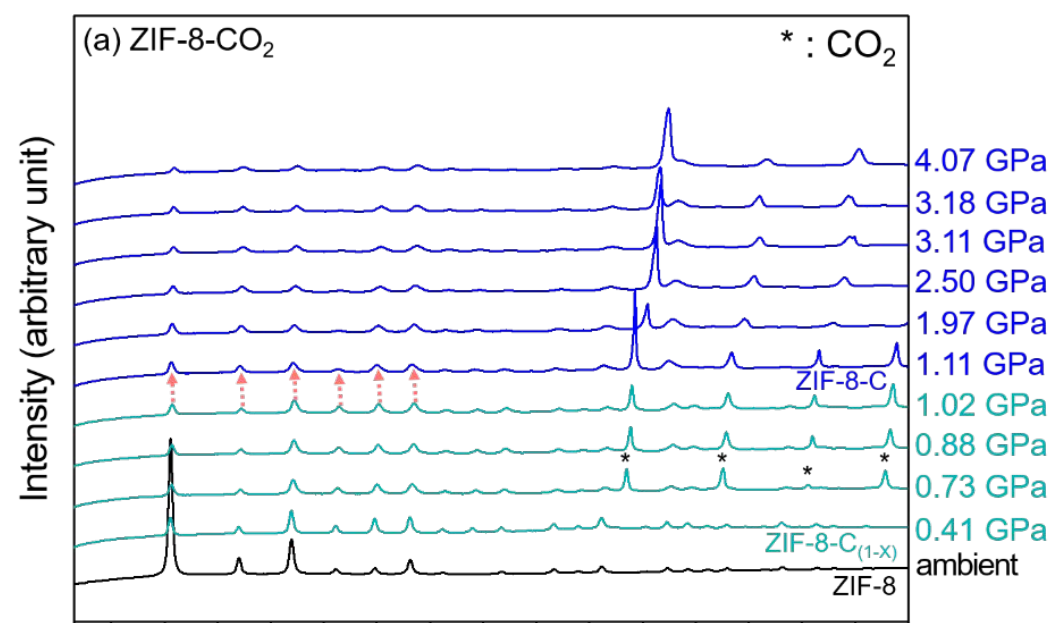

(b) ZIF-8-R22

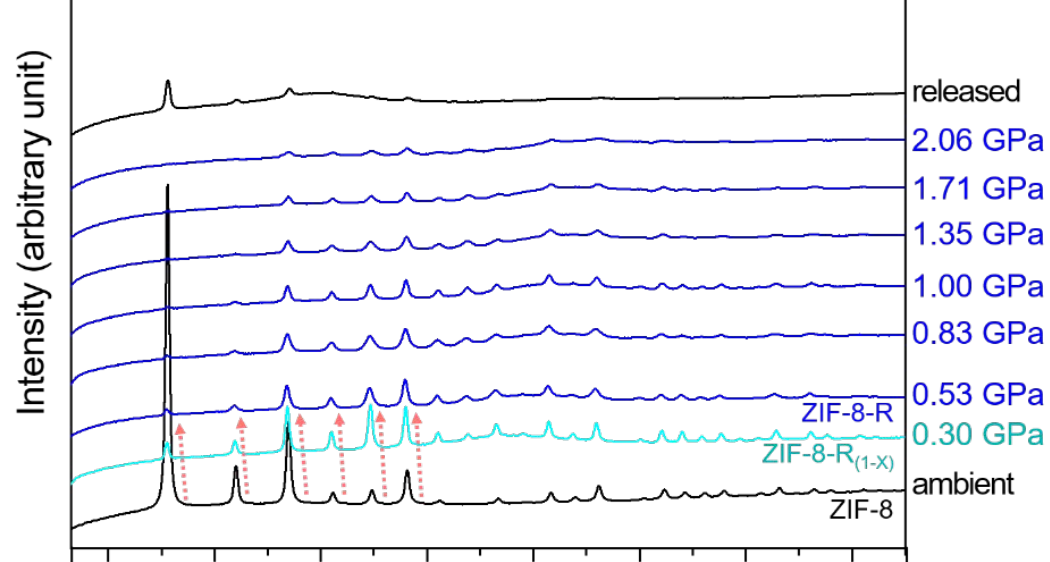

(c) ZIF-8-SF 6

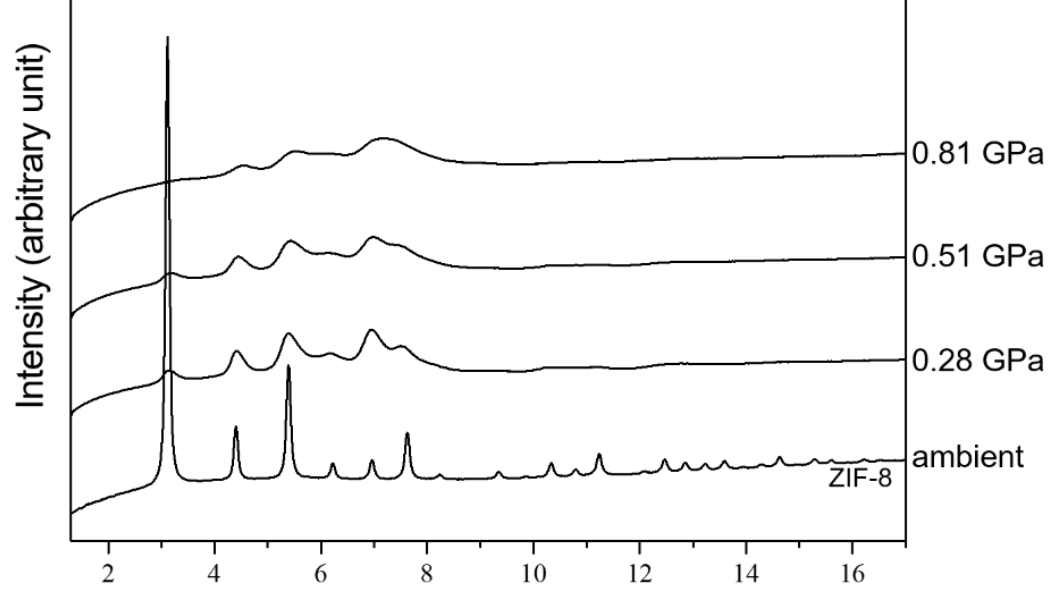

Two theta (degree)

Figure S1. Diffraction patterns of ZIF-8 at high pressures. ZIF-8-PII denotes a state where pressure-induced insertion (PII) of the different gases $\left(\mathrm{C}: \mathrm{CO}_{2}, \mathrm{R}: \mathrm{R} 22, \mathrm{~S}: \mathrm{SF}_{6}\right)$ is saturated, whereas the subscript (1-x) denotes a stage where PII is in progress. Note ZIF-8 compressed with $\mathrm{SF}_{6}$ shows peak broadening at relatively low pressures compared to those compressed with $\mathrm{CO}_{2}$ and $\mathrm{R} 22$. 

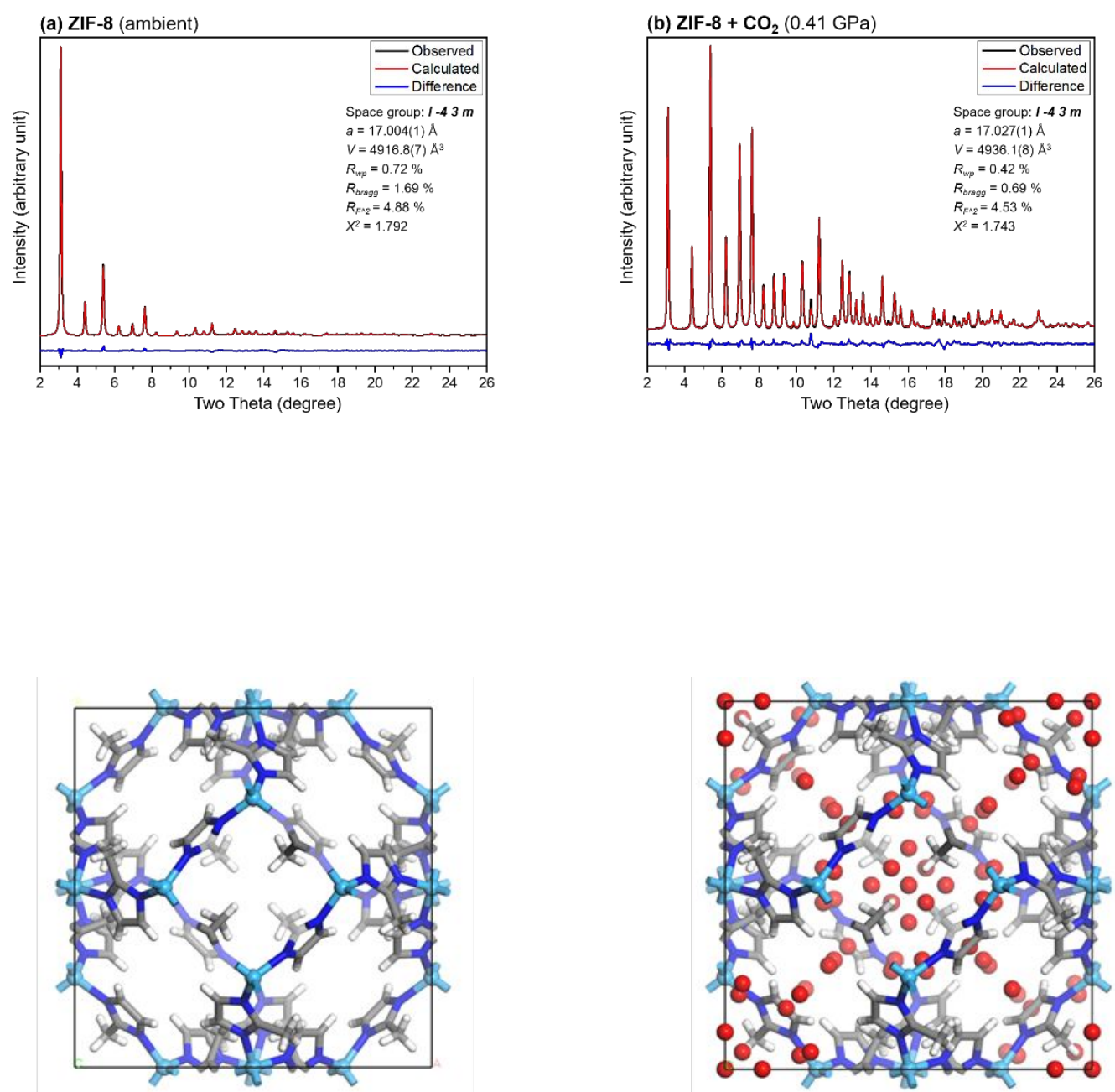

Figure S2. Rietveld fits of ZIF-8 at different pressures in $\mathrm{CO}_{2}$ pressure medium with respective structural models using oxygen dummy atoms. (a) ambient, (b) $4.1 \mathrm{kbar}$, (c) $7.3 \mathrm{kbar}$, (d) $8.8 \mathrm{kbar}$, (e) $10.2 \mathrm{kbar}$, (f) 11.1 kbar, (g) 19.7 kbar, (h) 25.0 kbar, (i) 31.1 kbar, (j) 31.8 kbar, (k) 40.7 kbar. 

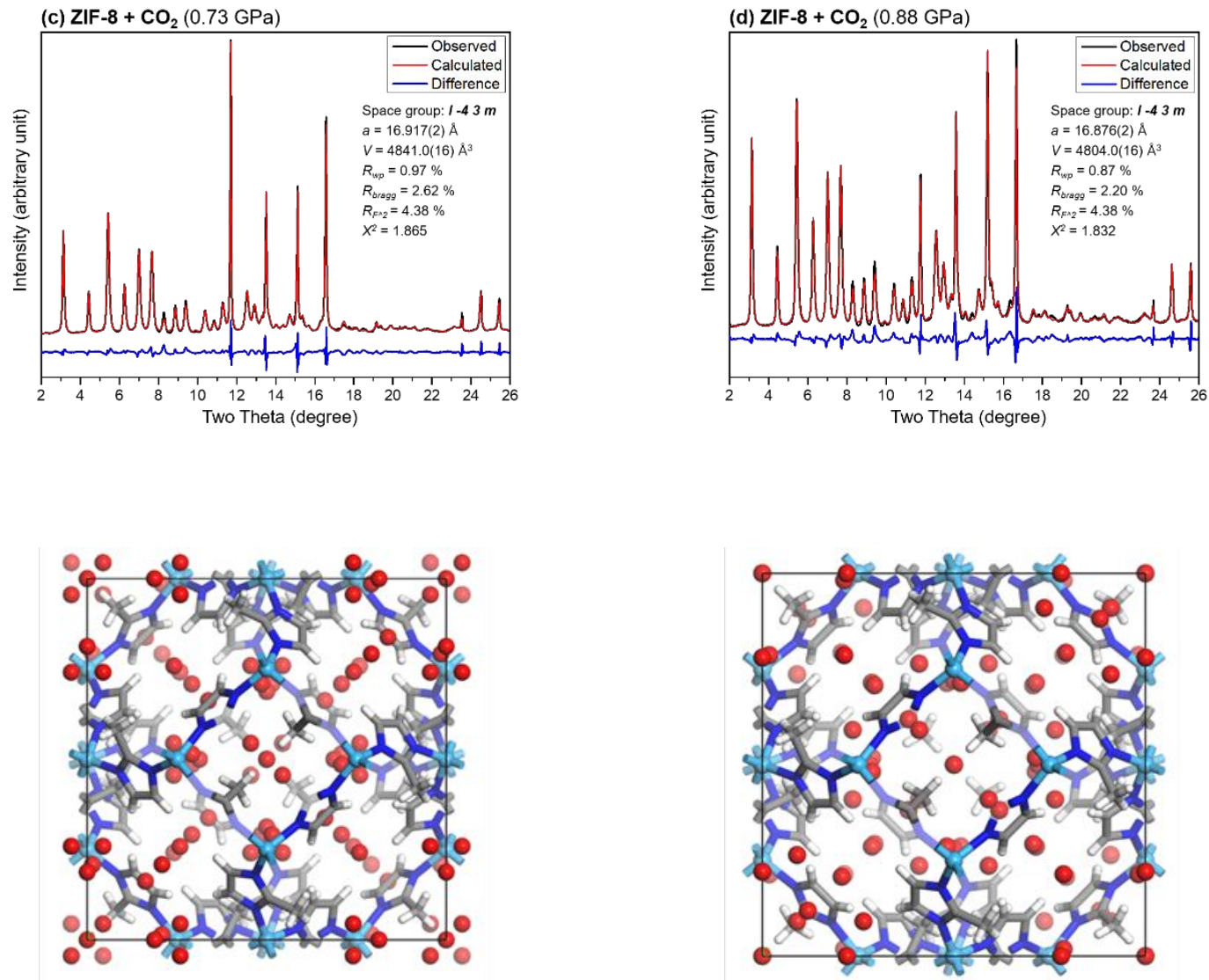

57 
67

68

69

70

71

72

73

(e) $\mathrm{ZIF}-8+\mathrm{CO}_{2}(1.02 \mathrm{GPa})$

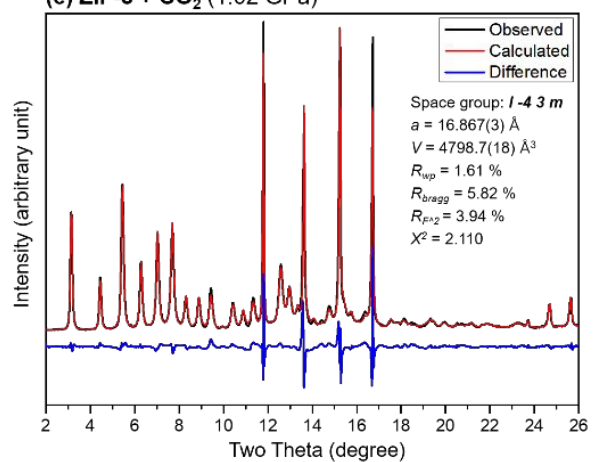

74

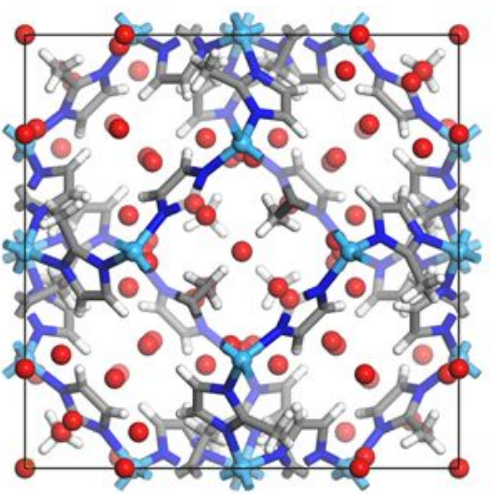

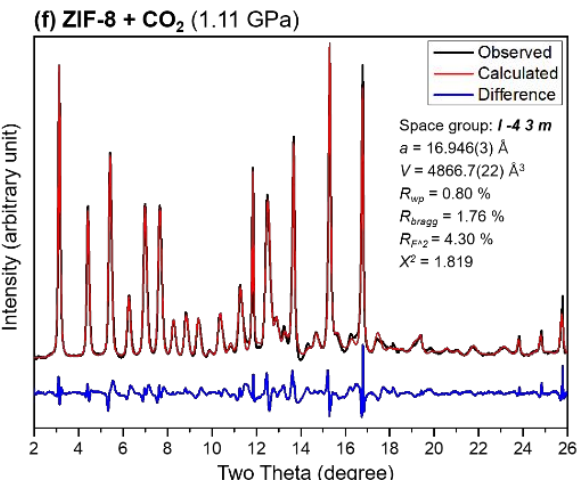

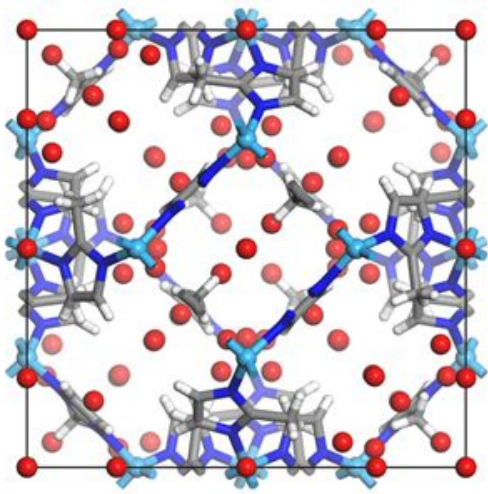


85

86

87

88

89

90

91

(g) ZIF-8 $+\mathrm{CO}_{2}(1.97 \mathrm{GPa})$

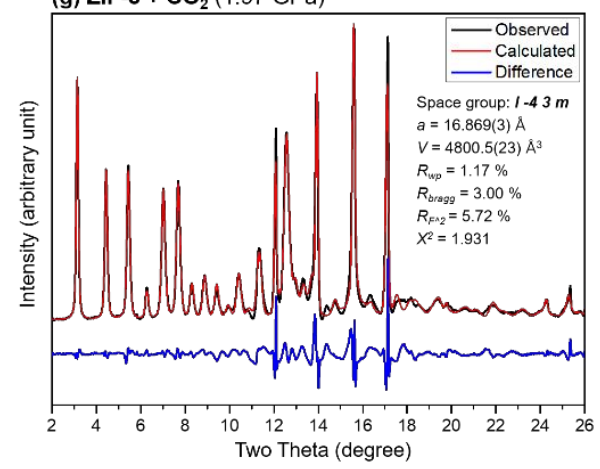

92

93

94

95

96

97

98

99

100

101

102

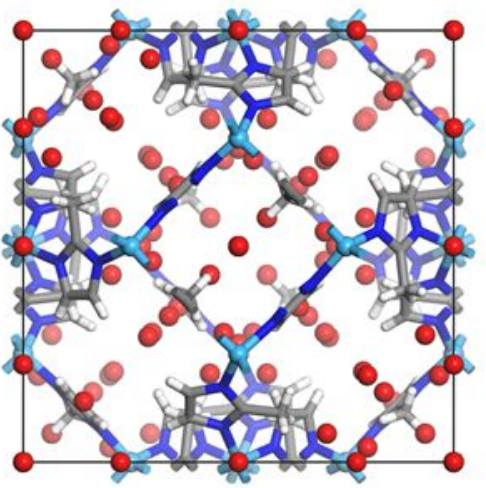

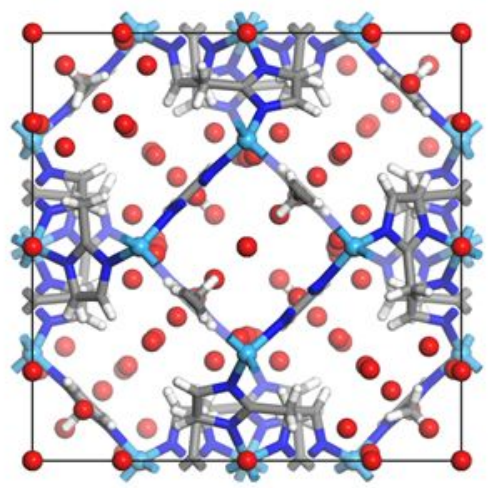

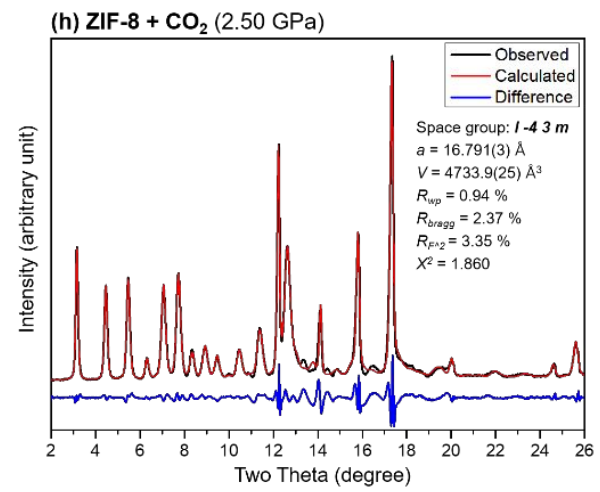

(h) ZIF-8 $+\mathrm{CO}_{2}(2.50 \mathrm{GPa})$ 

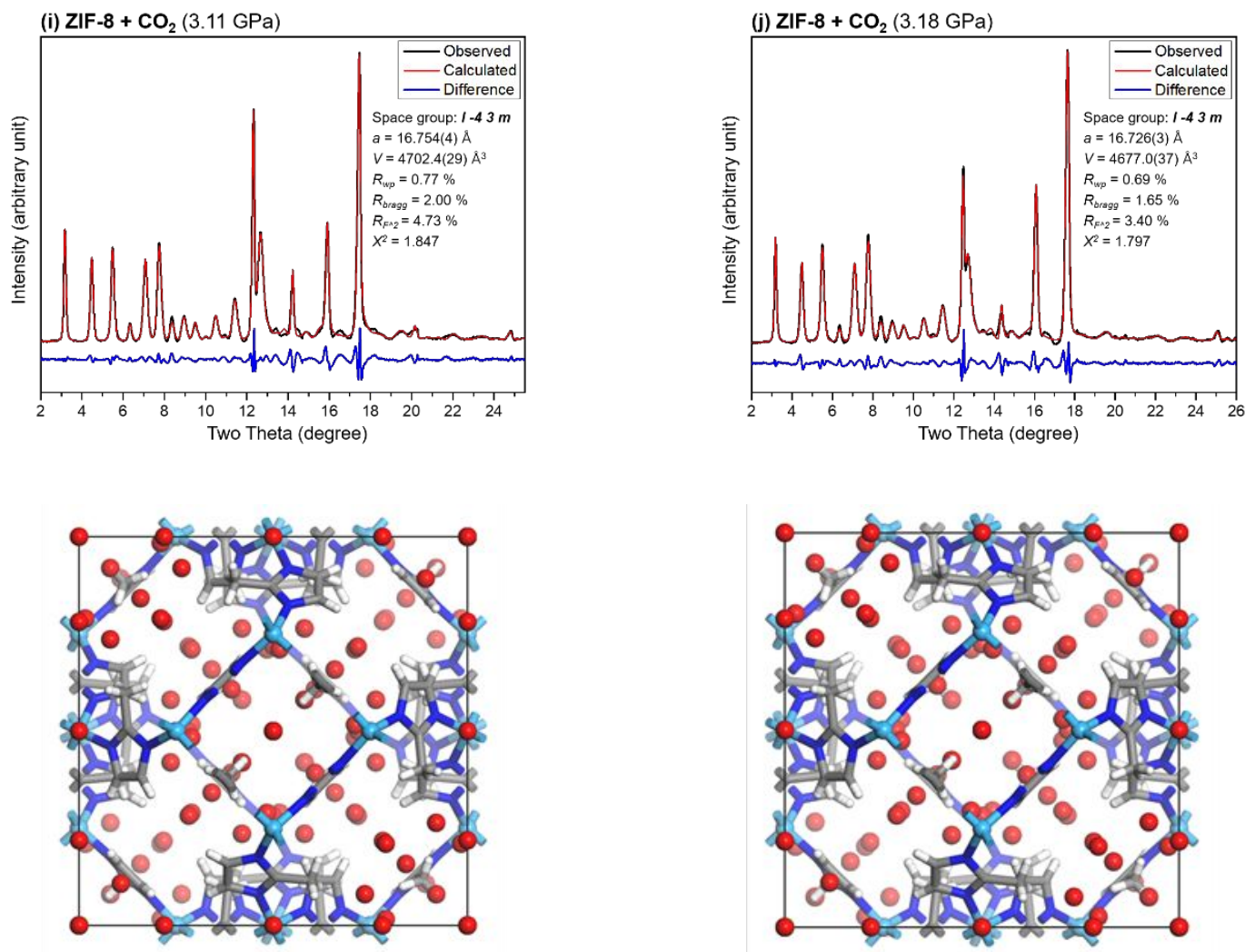
122

Figure S2. (continued)

123

124

125

126

127

128

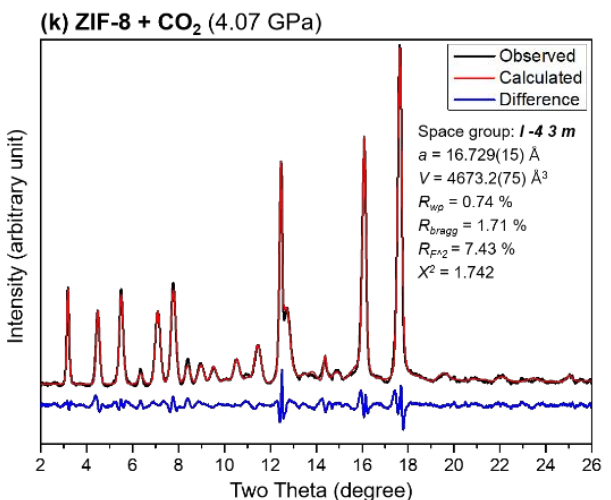

129

130

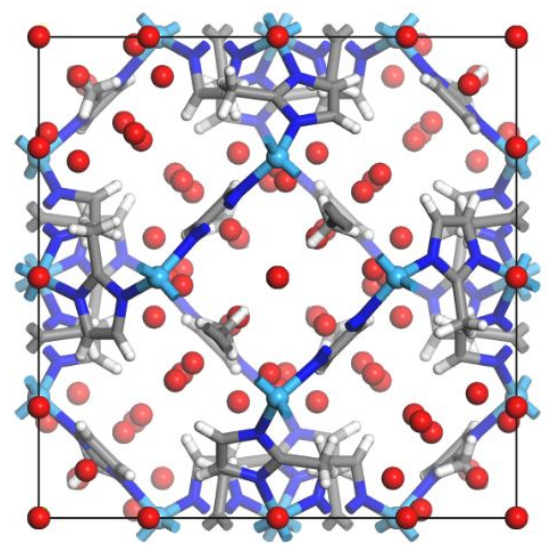

131 

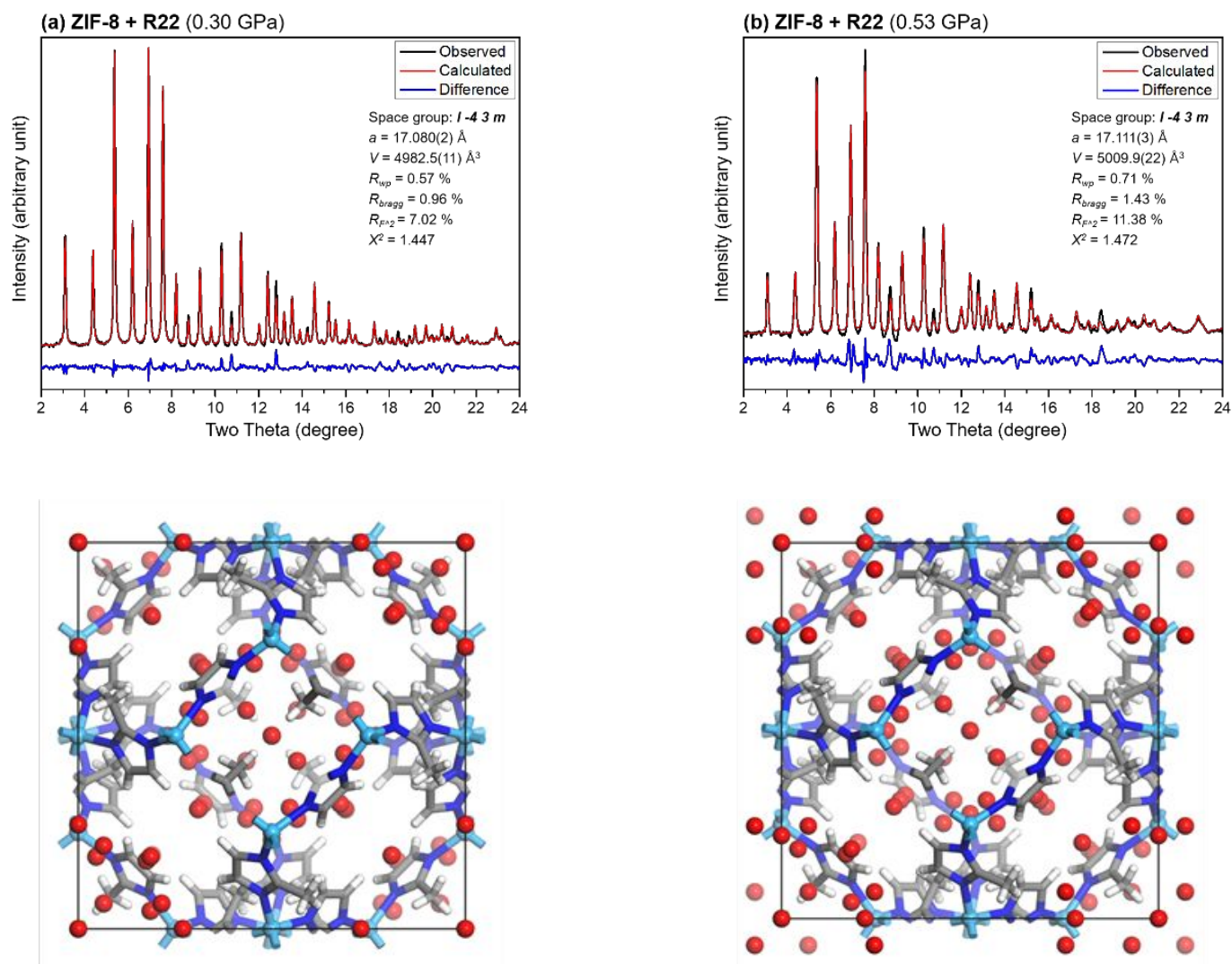

Figure S3. Rietveld fits of ZIF-8 at different pressures in R22 pressure medium with respective structural kbar, (g) 20.6 kbar. 

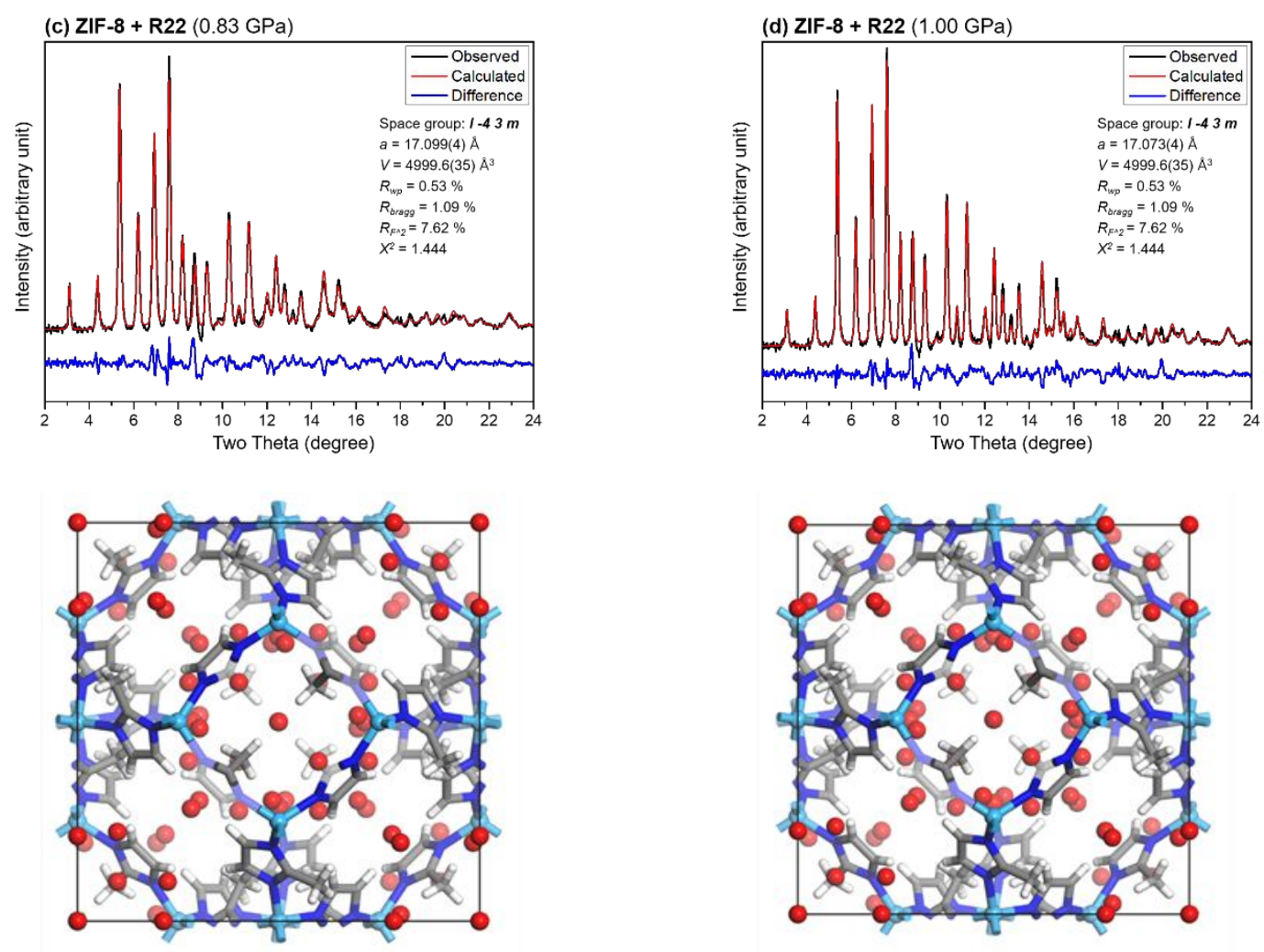

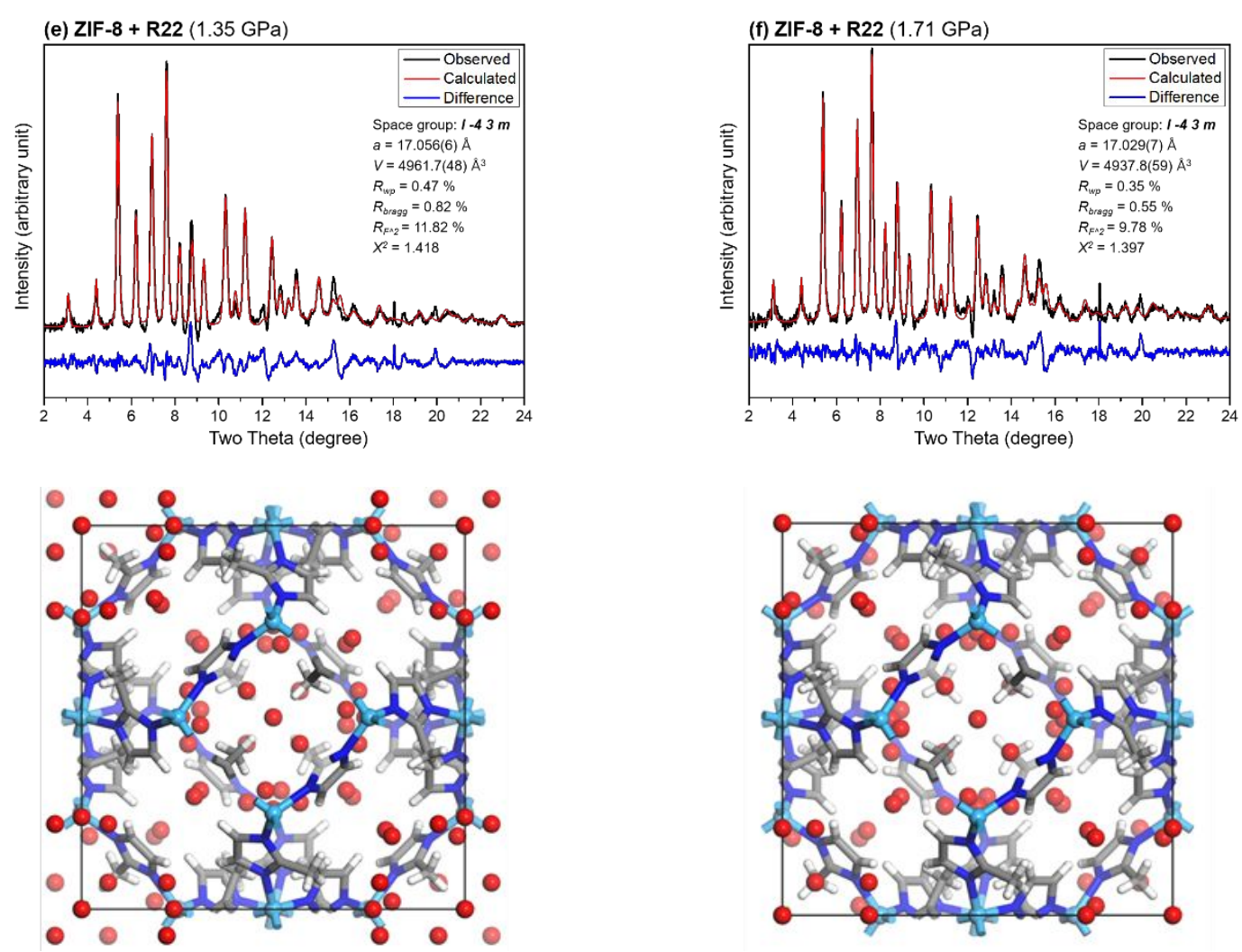

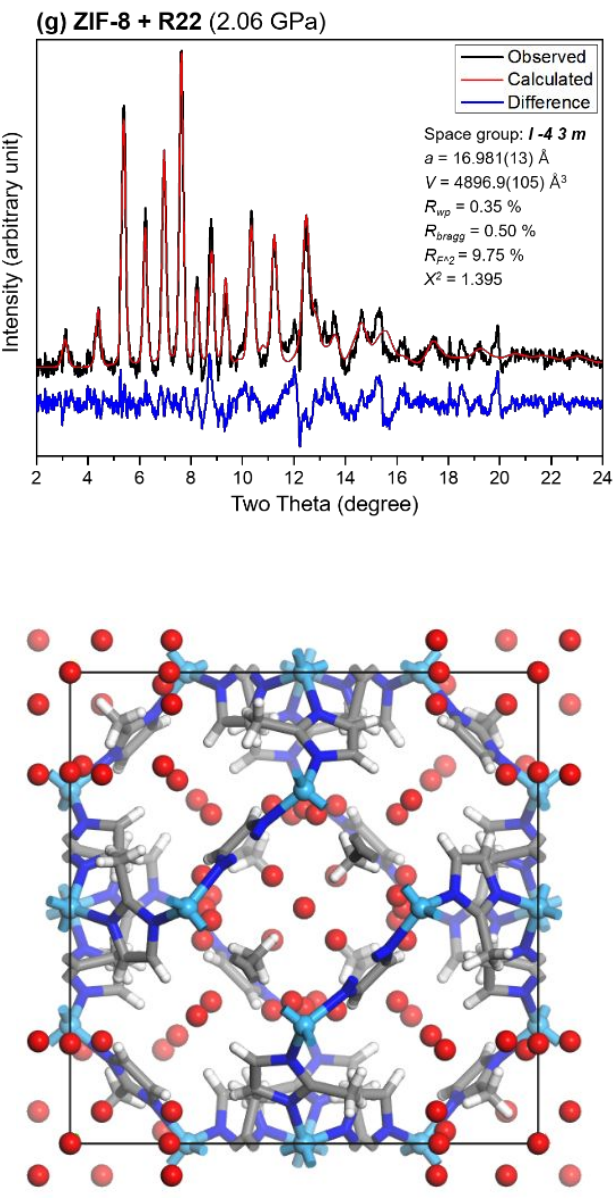
ZIF-8 + R22 (released)
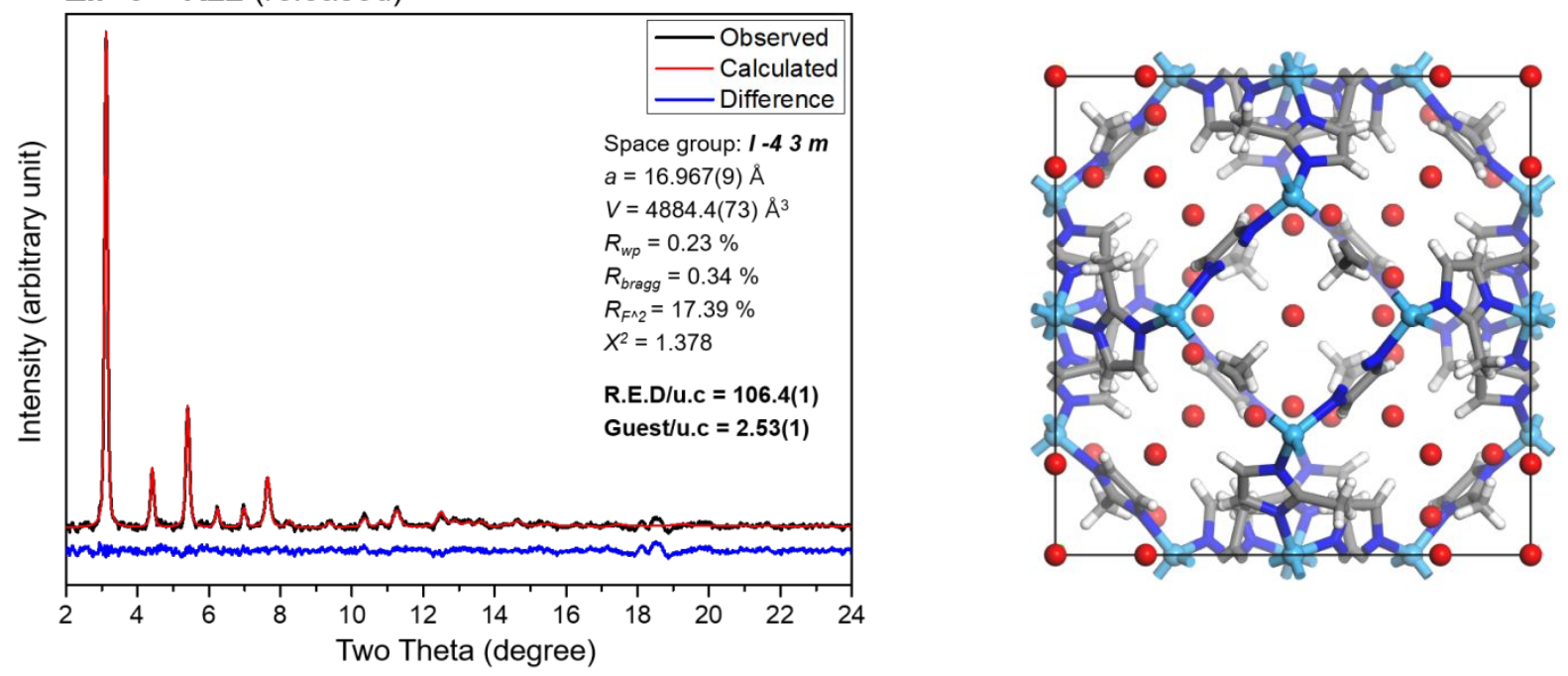

Figure S4. A Rietveld fit and structure model of pressure-released ZIF-8 after compression in the presence of

R22. Based on the residual electron density, the number of R22 molecules contained per unit cell is estimated to $2.53(1)$.

(a)

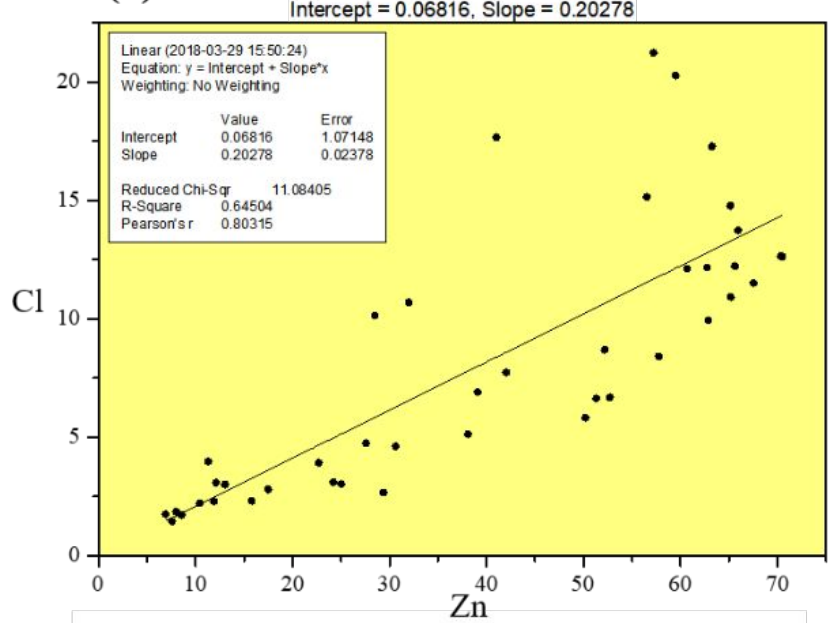

$\mathrm{Cl} / \mathrm{Zn}=0.2028(238)$

$\mathrm{Zn} /$ unit cell $=12$

$$
\begin{aligned}
\therefore \mathrm{Cl} / \text { unit cell } & =12 \times 0.2028(238) \\
& \approx \mathbf{2 . 4 3 ( 2 9 )}
\end{aligned}
$$

(b)

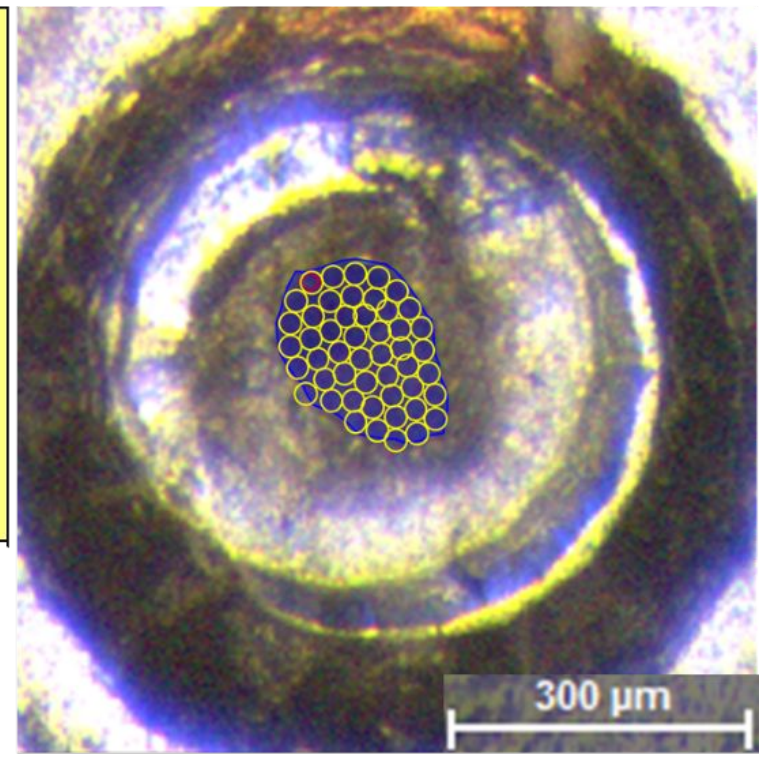

Figure S5. X-ray fluorescence spectroscopy of ZIF-8 after pressure release in the presence of R22. The number of residual R22 molecules are obtained from linear-fitting of the measured Cl/Zn counts. Total 49 points were scanned over the recovered ZIF-8 sample in the gasket hole, and 41 points that were not disturbed by stainless-steel gasket surrounding the sample were used for analysis. The $\mathrm{Cl}$ to $\mathrm{Zn}$ ratio at each point was linearly fitted to give 2.4(1) R22 molecules per unit cell, which agrees with the Rietveld result (Fig. S4). . (a) The result of the linear fit of $\mathrm{Cl} / \mathrm{Zn}$ counts. (b) An image of the recovered sample with yellow circles indicating XRF probed regions. 
(a) $\mathrm{ZIF}-8+\mathrm{CO}_{2}(0.73 \mathrm{GPa})$

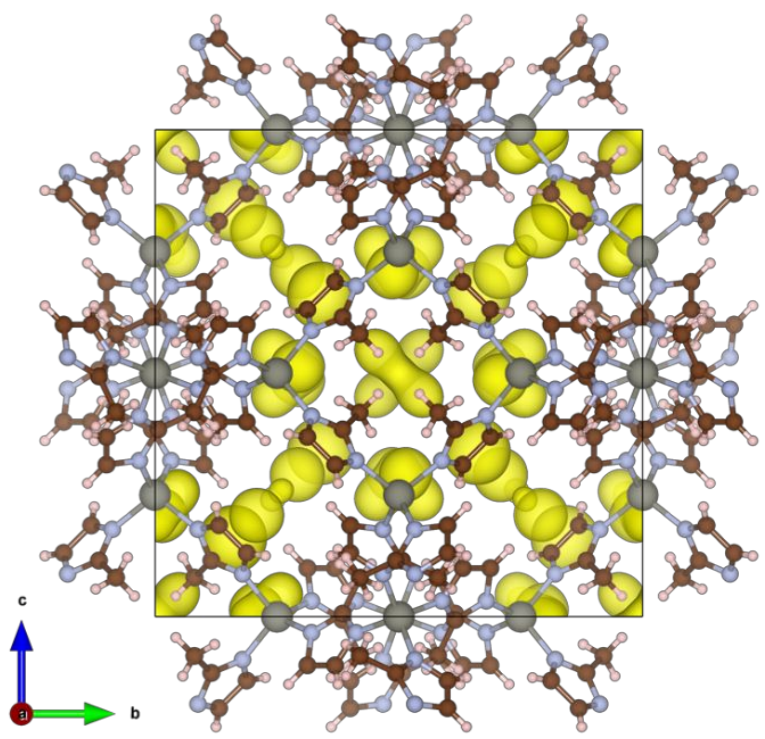

(b) $\mathrm{ZIF}-8+\mathrm{CO}_{2}(1.11 \mathrm{GPa})$

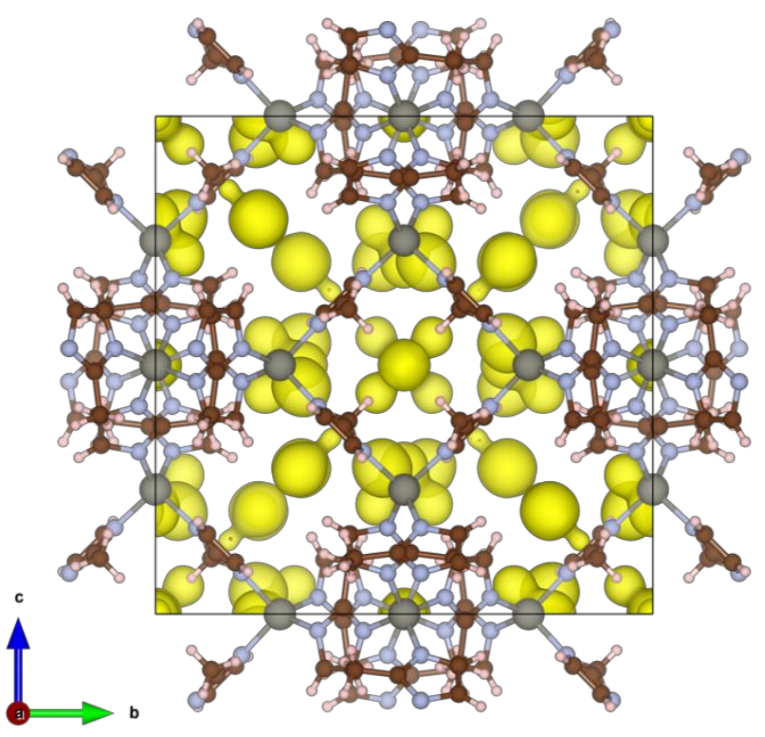

(c) ZIF-8 + R22 (0.83 GPa)

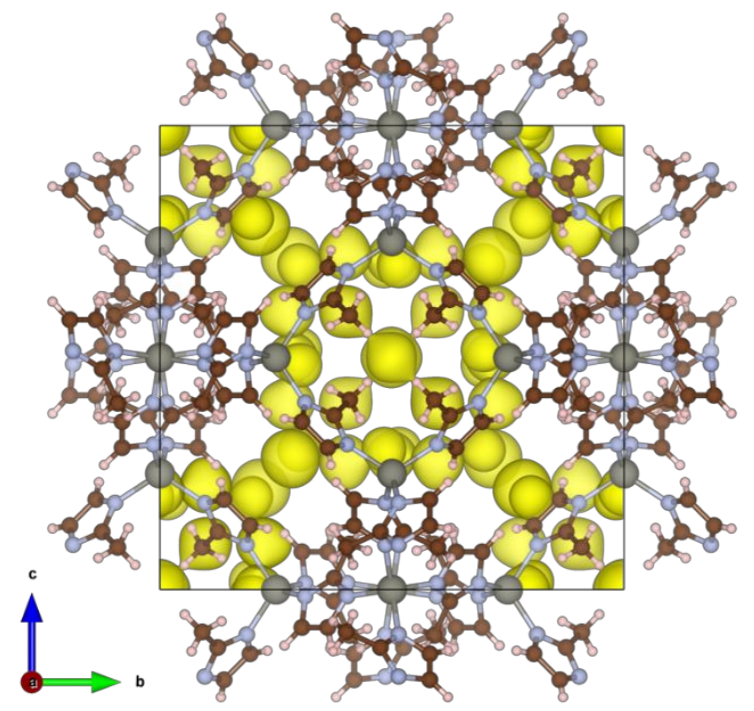

(d) ZIF-8 + R22 (2.06 GPa)

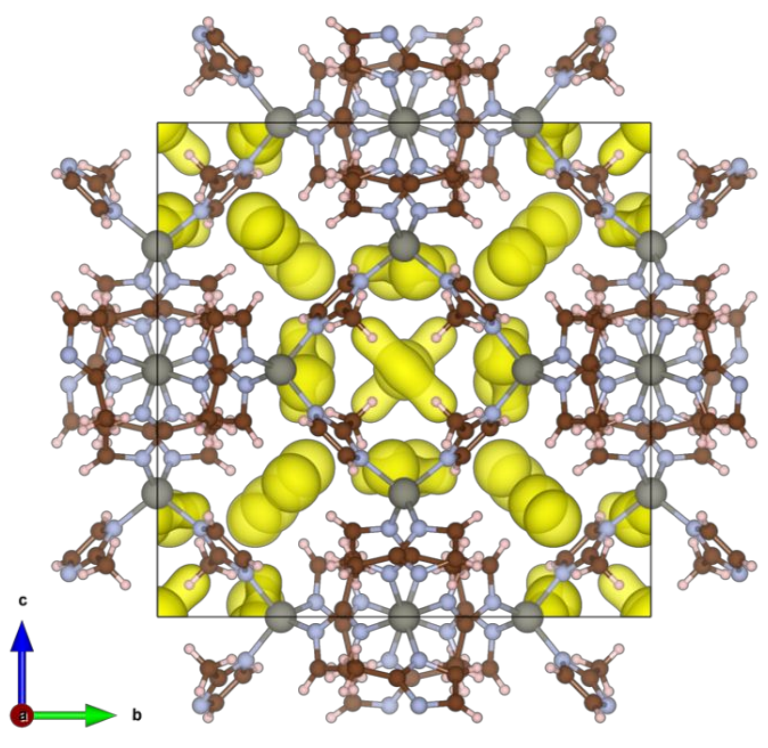

Figure S6. Electron density maps calculated from Rietveld refinement. Changes in ZIF-8 framework is along [100] direction. Note the incomplete pore opening of ZIF-8 compressed up to 20.6 kbar with R22, compared to ZIF-8 at $11.1 \mathrm{kbar}$ in $\mathrm{CO}_{2}$ PTM. 


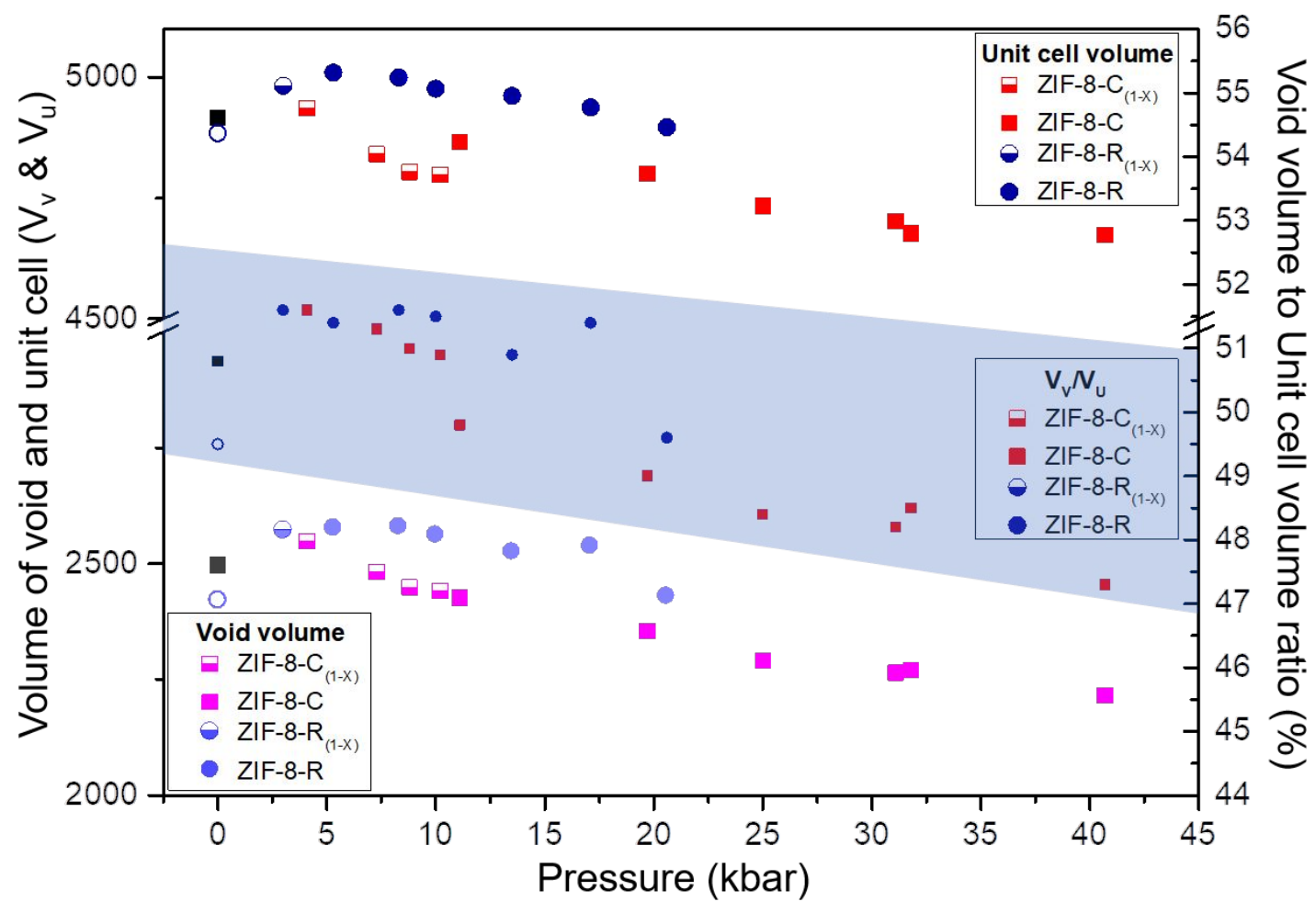

Figure S7. Unit cell and void volumes of ZIF-8 as a function of pressure. The data points within the blueshaded envelope show the ratio of void and unit cell volumes in percent. Note that the void to unit cell volume ratio decreases with increasing pressure. 


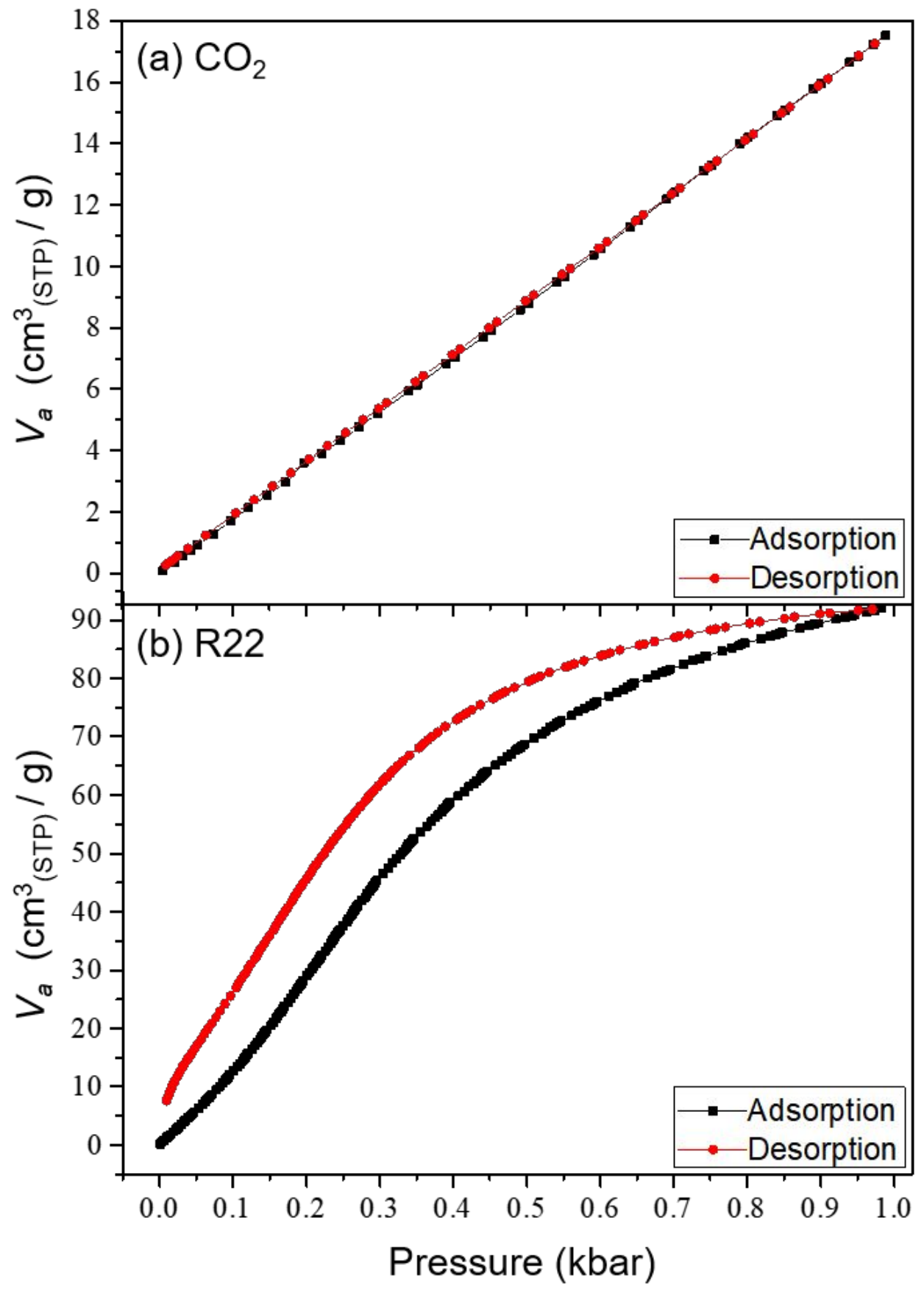

Figure S8. Adsorption/desorption isotherms of ZIF-8 under (a) $\mathrm{CO}_{2}$ and (b) R22 atmospheres. The amount adsorbed is estimated by the gas volume at standard conditions $\left(V_{a}\right)$. Note that R22 shows a higher degree of hysteresis and a certain amount of remnant gas after desorption, indicating its higher interaction with the framework compared to $\mathrm{CO}_{2}$. 
277 Table S1. Framework and pore data for ZIF-8 under $\mathrm{CO}_{2} \mathrm{PTM}$

\begin{tabular}{|c|c|c|c|c|c|c|}
\hline Pressure [kbar] & Unit cell Volume $\left(\mathrm{V}_{\mathrm{U}}\right)\left[\AA^{3}\right]$ & Void Volume $\left(\mathrm{V}_{\mathrm{V}}\right)\left[\AA^{3}\right]^{[\mathrm{a}]}$ & $\mathrm{V}_{\mathrm{V}} / \mathrm{V}_{\mathrm{U}}[\%]$ & Torsion angle $\left[{ }^{0}\right]^{[b]}$ & Electron Count $t^{[\mathrm{c}]}$ & $\mathrm{CO}_{2}$ molecules \\
\hline$\overline{0}$ & $4917(1)$ & 2539 & 50.8 & 60.0 & 0 & 0 \\
\hline 4.1 & $4936(1)$ & 2571 & 51.6 & 58.9 & 882 & 40.1 \\
\hline 7.3 & $4841(2)$ & 2500 & 51.3 & 60.2 & 1003 & 45.6 \\
\hline 8.8 & $4804(2)$ & 2445 & 51.0 & 60.2 & 1042 & 47.4 \\
\hline 10.2 & $4799(2)$ & 2468 & 50.9 & 60.3 & 1072 & 48.7 \\
\hline 11.1 & $4867(3)$ & 2524 & 49.8 & 84.4 & 1167 & 53.0 \\
\hline 19.7 & $4801(3)$ & 2498 & 49.0 & 85.1 & 1230 & 55.9 \\
\hline 25.0 & 4734(3) & 2410 & 48.4 & 86.8 & 1231 & 56.0 \\
\hline 31.1 & $4702(3)$ & 2388 & 48.2 & 86.5 & 1232 & 56.0 \\
\hline 31.8 & $4677(4)$ & 2356 & 48.5 & 85.5 & 1233 & 56.1 \\
\hline 40.7 & $4673(8)$ & 2350 & 47.3 & 83.2 & 1234 & 56.1 \\
\hline
\end{tabular}

[a] Calculated using Mercury CSD 3.10.3 with a probe radius of $1.2 \AA . \quad$ [b] The angle between mean plane of imidazolate and crystallographic (100) plane. [c] Obtained from Rietveld refinement.

Table S2. Framework and pore data for ZIF-8 under R22 PTM

\begin{tabular}{|c|c|c|c|c|c|c|}
\hline Pressure [kbar] & Unit cell Volume $\left(\mathrm{V}_{\mathrm{U}}\right)\left[\AA^{3}\right]$ & Void Volume $\left(\mathrm{V}_{\mathrm{V}}\right)\left[\AA^{3}\right]^{[\mathrm{a}]}$ & $\mathrm{V}_{\mathrm{V}} / \mathrm{V}_{\mathrm{U}}[\%]$ & Torsion angle $\left[{ }^{0}\right]^{[b]}$ & Electron Count ${ }^{[\mathrm{c}]}$ & R22 molecules \\
\hline$\overline{0}$ & $4917(1)$ & 2539 & 50.8 & 66.0 & 0 & 0 \\
\hline 3.0 & 4983(2) & 2571 & 51.6 & 61.2 & 926 & 22.0 \\
\hline 5.3 & $5010(3)$ & 2576 & 51.4 & 62.3 & 1053 & 25.1 \\
\hline 8.3 & $5000(4)$ & 2579 & 51.6 & 61.6 & 1068 & 25.4 \\
\hline 10.0 & 4977(3) & 2561 & 51.5 & 59.8 & 1070 & 25.5 \\
\hline 13.5 & $4962(5)$ & 2524 & 50.9 & 67.1 & 1084 & 25.8 \\
\hline 17.1 & $4938(6)$ & 2537 & 51.4 & 61.0 & 1083 & 25.8 \\
\hline 20.6 & $4897(11)$ & 2428 & 49.6 & 76.1 & 1084 & 25.8 \\
\hline 0 (released) & $4884(8)$ & 2419 & 49.5 & 75.8 & 106 & 2.53 \\
\hline
\end{tabular}

[a] Calculated using Mercury CSD 3.10.3 with a probe radius of $1.2 \AA . \quad$ [b] The angle between mean plane of imidazolate and crystallographic (100) plane. 